\title{
Modélisation des familles de produits : État de l'art
}

\author{
Bruno AGARD ${ }^{\mathrm{a}}$
}

Département de Mathématiques et de Génie Industriel, École polytechnique de Montréal, C.P. 6079, succ. Centre-ville, Montréal (Québec), H3C3A7, Canada

Reçu le 4 décembre 2002, accepté le 5 décembre 2003

Résumé - Dans le cas d'un produit unique et non évolutif, on ne rencontre que très peu de difficultés à la représentation des données le concernant. Cependant dans un contexte de forte diversité, il s'avère impossible de représenter explicitement chaque produit par une nomenclature spécifique. L'article présenté ici porte sur la modélisation des familles de produits. Afin de représenter une grande diversité de produits la structuration des données techniques est abordée par la codification des objets techniques et leur classification. Les modèles de représentation basés sur les liens sont exposés. Les modèles génériques de représentation de la diversité sont montrés avec les modèles de configurations. Finalement les principaux points de consensus sont relevés ainsi que les manques quand à la dynamique de versionnement et à la représentation du processus.

Mots clés : Modélisation / familles de produits / codification / classification / liens / modèles génériques / STEP

\begin{abstract}
Modeling product families: State of the art. There are no major difficulties in the representation of a single and nonevolutionary product. However in a context of strong diversity, it is impossible to represent explicitly each product by a specific bill of material. This paper relates to the modelling of product families. In order to represent a large diversity of products the structuring of technical data is presented through the coding of the technical objects and their classification. Models of representation based on links are exposed. Generic models and configuration models are covered. Finally the main points of consensus are emphasized as well as the lacks regarding dynamics of the model and lacks in the process representation.
\end{abstract}

Key words: Modeling / product families / codification / classification / links / generic modeling / STEP

\section{Introduction}

Dans le cas d'un produit unique et non évolutif, on ne rencontre que très peu de difficultés à la représentation des données le concernant. Cependant dans un contexte de forte diversité, il s'avère impossible de représenter explicitement chaque produit par une nomenclature spécifique.

Une solution apportée par les industriels est d'utiliser des familles de produits qui permettent un certain degré de standardisation, et laissent encore de la flexibilité au produit pour pouvoir représenter chaque individu. Ces familles de produits se divisent ensuite en sousfamilles (produits) de manière à mieux cibler un marché segmenté, ensuite chaque produit de la sous-famille

\footnotetext{
a Auteur correspondant : bruno.agard@polymtl.ca
}

(instance) peut être personnalisé selon les besoins et désirs de chaque client.

Une modélisation basée sur un partage suivant différents niveaux de la représentation du produit est présentée figure 1. Les niveaux considérés sont : la famille de produit, le type de produit et l'instance de produit qui au niveau des nomenclatures correspondent à nomenclature générique, nomenclature spécifique et nomenclature instanciée, chacune d'entre elles se déclinant en plusieurs vues (fonctionnelle, structurelle, maintenance, ...).

- la nomenclature générique : contient les concepts de base, au niveau des nomenclatures, il s'agit du plus haut degré d'abstraction. Ce type de nomenclature contient les familles de produits. Par exemple, la famille des Peugeot 306, la famille des Citroën ZX, la famille des ordinateurs portables, ...

- la nomenclature spécifique : dans ces nomenclatures, sont rangés les types de produit. Nous nous situons 


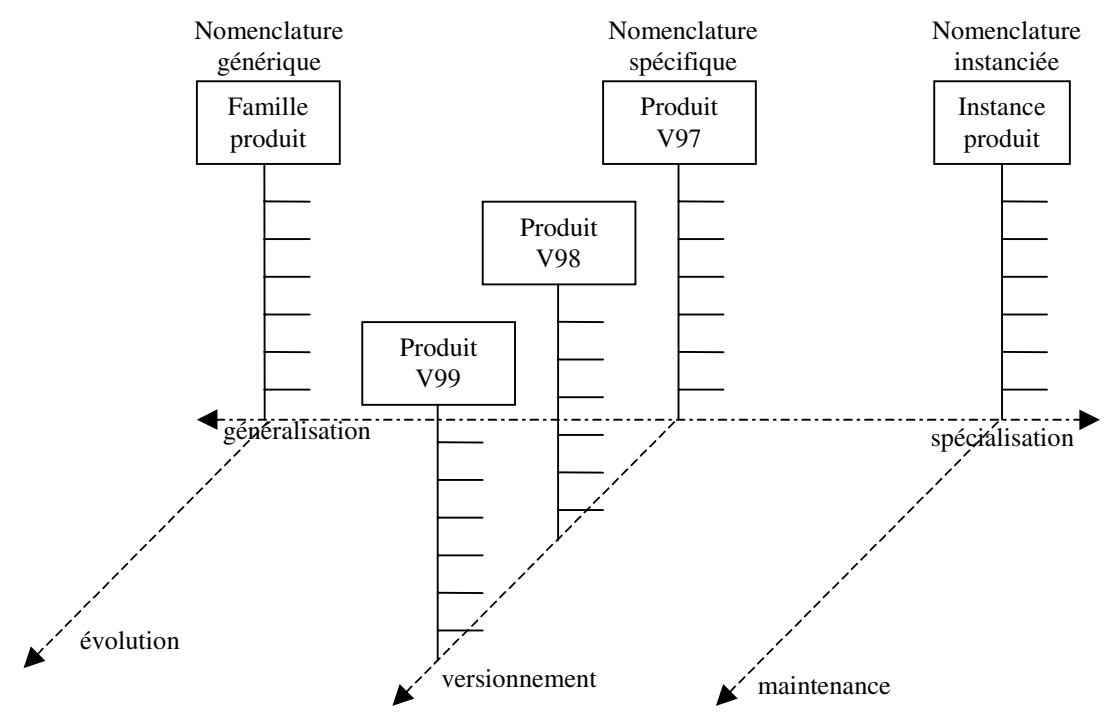

Fig. 1. Différents niveaux de nomenclatures.

donc à un niveau d'abstraction inférieur. Il s'agit à ce niveau des données relatives aux modèles de produit : les Peugeot 306 XT, les Citroën ZX Avantage, ...

- la nomenclature instanciée : ce dernier modèle de nomenclature est celui de plus bas niveau, il contient les données spécifiques à une instance de produit en particulier. On peut trouver par exemple la nomenclature de la voiture Citroën ZX Avantage immatriculée : 702 AZN 38.

Ces trois types de nomenclatures ne se situent pas au même niveau de la représentation des données techniques pour un même produit. Elles ne permettent pas de représenter le même type de données, elles cœxistent et se partagent la représentation du produit. Il convient donc de rattacher les informations au bon niveau. Ces différents niveaux de représentation du produit proviennent de différents besoins au niveau de la conception :

- Par exemple, la nomenclature générique peut servir en conception pour classer les données relatives aux familles de produits.

- De même, la nomenclature spécifique, permet de structurer les données relatives à la conception d'un type de produit en particulier.

- La nomenclature instanciée, permet quant à elle de suivre l'évolution d'un produit en particulier, cette nomenclature est très utile en maintenance par exemple.

Simultanément, toujours au niveau de la conception, quel que soit le niveau (famille, produit ou instance), il existe suivant un autre axe, un versionnement des constituants et des liens qui permet de caractériser les différentes évolutions du produit.

Par ailleurs, il existe dans l'entreprise différentes formes de représentations du produit (représentations implicites ou explicites de la structure du produit) parmi lesquelles :

- les analyses fonctionnelles,
- les plans, qui contiennent en général les données produit ainsi qu'une structure de produit implicite,

- les nomenclatures, qui contiennent la structure des composants,

- les dessins d'assemblages, qui fournissent une vue structurelle de la décomposition physique,

- la documentation de maintenance,

- les catalogues de produits, ...

Pour une entreprise qui n'est pas totalement intégrée, les types de documents actuels rendent difficile la comparaison entre les variantes de produit, par exemple une même pièce peut avoir différentes codifications selon les représentations. Dans les cas de conception avec réutilisation de solutions existantes, il y a un manque de documents compréhensibles de l'ancienne conception (références croisées entre les documents).

Différents modèles ont donc été proposés dans la littérature. Ils se retrouvent en général sous l'appellation de familles de produit. Ces modèles permettent de représenter des produits génériques ou des composants génériques.

\section{Structuration des données techniques}

Différentes propositions de structuration des données techniques tirées de la littérature sont présentées ici, en commençant par la codification des objets techniques, puis par la classification.

\subsection{Codification des objets techniques}

D'après Maurino [1], le principe de base de la codification est que tout objet technique est identifié par une référence unique. Cette référence porte suivant les entreprises les noms de code article, numéro de nomenclature, part-number, référence composant, ... 
Il existe de très nombreux systèmes de codification des objets techniques qui dépendent des entreprises, des secteurs d'entreprise, des projets. Il est cependant possible de les ranger dans quelques grandes classes de codification.

\subsubsection{La codification unique}

Dans ce type de codification, la référence à l'objet technique est unique. Un objet technique entrant dans la composition de plusieurs produits possède donc une seule référence, commune à tous les cas d'emploi de l'objet.

Intérêts de cette unicité :

- éviter la duplication des informations de définition de l'objet technique (éviter les risques de non-intégrité des données dupliquées),

- supporter les efforts de standardisation des produits : il est aisé d'utiliser un produit déjà existant en réutilisant simplement sa codification,

- effectuer le regroupement des besoins en matières, composants, et sous-ensembles entrant dans la composition des différents produits inscrits au plan directeur, selon le principe $\mathrm{MRP}^{1}$,

- optimiser la gestion des stocks.

\subsubsection{La codification significative}

Le principe de codification significative s'oppose aux méthodes de codification unique de l'objet technique. Elles sont utilisées, en l'absence de systèmes de $\mathrm{GPAO}^{2}$, pour gérer manuellement la production. La codification d'un objet n'est pas seulement une référence à celui-ci, mais porte en plus une sémantique qui précise le cas d'emploi. Une codification significative se compose d'un ensemble ordonné de références.

La référence attribuée à un article permet :

- d'identifier l'article lui-même (suffixe de la codification),

- d'identifier l'ensemble dans lequel intervient l'article (racine de la référence).

Comme le montre Maurino [1] dans l'exemple donné en tableau 1, la codification d'une pièce précise à quels ensembles (sous-ensembles) de quels produits appartient la pièce.

Cependant, quelques inconvénients sont à l'origine de la disparition de ce type de codification :

- il existe autant de références que de cas d'emploi. Si le même produit est utilisé dans différents sousensembles, il aura différentes codifications,

- elle est un obstacle à la standardisation. La réutilisation de composants dans différents ensembles nécessite la création de nouvelles références,

\footnotetext{
${ }^{1}$ MRP : Material Ressource Planning [2,3].

${ }^{2}$ GPAO : Gestion de Production Assistée par Ordinateur $[2,3]$.
}

Tableau 1. Exemple de codification significative.

\begin{tabular}{lr}
\hline Article & Référence \\
\hline produit & 130 \\
ensemble & 130220 \\
& 130270 \\
sous ensemble & 130270500 \\
& 130270580 \\
pièce primaire & 130270581 \\
\hline
\end{tabular}

- il n'y a pas d'optimisation des stocks. En effet, il est difficile de retrouver toutes les références d'un même produit de manière à gérer tous les flux d'entrée et de sortie du composant entrant dans différents ensembles.

Un avantage par rapport à la codification unique est la gestion des liens unissant un ensemble et les articles entrant dans sa composition.

\subsubsection{Les méthodes mnémotechniques}

Elles sont utilisées notamment dans la chimie organique qui dispose d'un moyen très efficace d'organisation de l'information et des données : il s'agit de la molécule, représentée sous forme de dessin.

Le caractère unique de la molécule permet de lui associer de façon biunivoque un numéro qui donne accès à toute l'information concernant cette molécule. Le numéro sert de manière transparente pour l'utilisateur, de clef d'accès aux données et aux informations.

Hors du domaine de la chimie organique, on perd le bénéfice de cette cohérence interne, l'information est alors structurée par sujets, définis de façon plus ou moins arbitraires et variables dans le temps, ceci nécessite l'existence d'index et de thésaurus, complexes à mettre en place et à maintenir.

L'avantage des procédés mnémotechniques est l'accessibilité à l'information.

\subsubsection{La codification des exemplaires}

Lorsque l'on utilise plusieurs exemplaires de la même référence article, il peut sembler utile d'en tenir compte dans la codification de manière à représenter cette similitude. Pour cela il existe deux types de codification :

1. On utilise la référence article à laquelle on ajoute un numéro de série.

Ex : 130270580 (code article) + 256 (numéro de série) $=130270580-256$

Cependant cette codification reprend en partie le principe de la codification significative, ce qui pose quelques problèmes en cas de modification.

2. Une autre solution est de créer des références non significatives et de gérer des liens comme représenté sur la figure 2. On sait ainsi que les références 65421, 98165 et 1987154 sont des exemplaires de l'article 123456. 


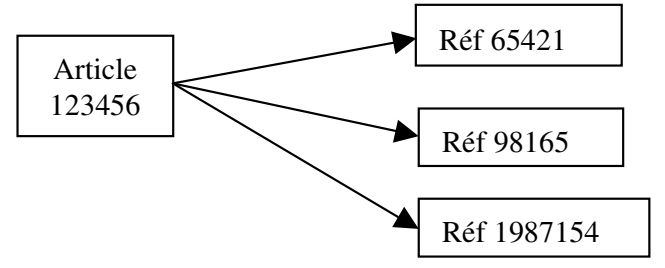

Fig. 2. Codification des exemplaires.

Cette méthode est plus rigoureuse et permet de gérer les modifications, elle est en revanche plus complexe à mettre en ouvre.

\subsection{Classification des objets techniques}

Le but de la classification est de créer des ensembles (de produits, de composants, ...) partageant certaines caractéristiques. Il est possible de distinguer trois propositions : les familles d'objets, les bibliothèques de composants et la classification à facettes.

\subsubsection{Familles d'objets par héritage}

En associant les points communs à différents articles, les objets techniques peuvent être regroupés en familles. Selon les critères de comparaison des articles, il est possible d'obtenir différentes familles (fonctionnelles, technologiques, ...) que l'on peut classer en arborescences [1].

La structure arborescente des familles est telle que plus on descend dans l'arbre, plus les critères sont précis et inversement plus on monte plus les critères sont génériques (Fig. 3).

Les caractéristiques communes sont attachées aux familles et par héritage aux objets techniques. Ces caractéristiques participent à la définition des familles, et à l'identification précise de chaque objet dans une famille déterminée. Une famille peut être générée à partir d'une autre :

- par adjonction de caractéristiques (spécialisation),

- par la définition de valeurs ou de fourchettes de valeurs de certaines caractéristiques (instanciation).

Ceci est représenté sur la figure 4.

Les caractéristiques se transmettent de père en fils en suivant l'arborescence des familles et des objets techniques, suivant un phénomène d'héritage. Il existe deux types d'héritage :

- l'héritage simple (cf. Fig. 3), la famille fille hérite les caractéristiques de la famille mère,

- l'héritage multiple (cf. Fig. 5), la famille fille hérite les caractéristiques des familles parentes.

Ces idées sont reprises dans les normes.
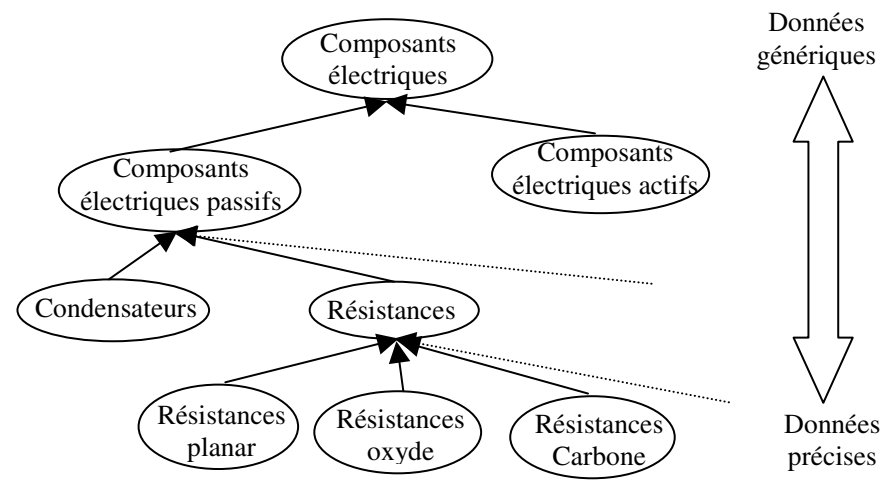

Fig. 3. Les familles d'objets techniques.

\subsubsection{Bibliothèques de composants}

Il existe différents modèles de classification des composants, qui restent tout de même très proches les uns des autres. Les modèles sont statiques, les familles de pièces sont décrites et définies en leur appliquant des propriétés à un certain niveau de hiérarchie. Il y a donc une définition simultanée des propriétés et des familles, dans une Approche Orientée Objet.

Dietz et Ort dans [4] ont comparés les normes DIN 4000 et ISO 13584.

Approche DIN 4000 Il s'agit de rassembler des objets standardisés ou non, physiques ou non, similaires dans un sens. Pour les décrire, les différents termes suivant sont utilisés : Paramètres, Objets, Groupes d'objets et Liste de paramètres d'objets. Les paramètres sont utilisés pour caractériser des groupes d'objets et sont rassemblés dans une liste de paramètres d'objets. Il faut alors appliquer une valeur à un des paramètres pour décrire un objet de ce groupe d'objets.

Approche ISO 13584 Il s'agit ici de Modélisation Orientée Objet. Le vocabulaire utilisé n'est donc plus le même : Propriétés (caractérisent les familles, simples et génériques), Pièces, Famille simple, Famille générique et Hiérarchie. Les familles sont structurées en hiérarchies en utilisant l'héritage des caractéristiques des familles parentes. Les familles sont caractérisées par des propriétés.

Dans les deux cas, les méthodes proposées sont des méthodes a posteriori, qui fonctionnent d'après le schéma suivant :

1. sélection de l'ensemble des produits concernés (domaine d'étude),

2. regroupement en fonction de leurs points communs,

3. création d'une classe de produit pour chaque ensemble.

Les modèles de représentations s'appuient ensuite soit sur une architecture « une sorte de $»$ : DIN 4000, soit sur un modèle objet permettant de définir des hiérarchies et des héritages de propriétés : ISO 13584. 


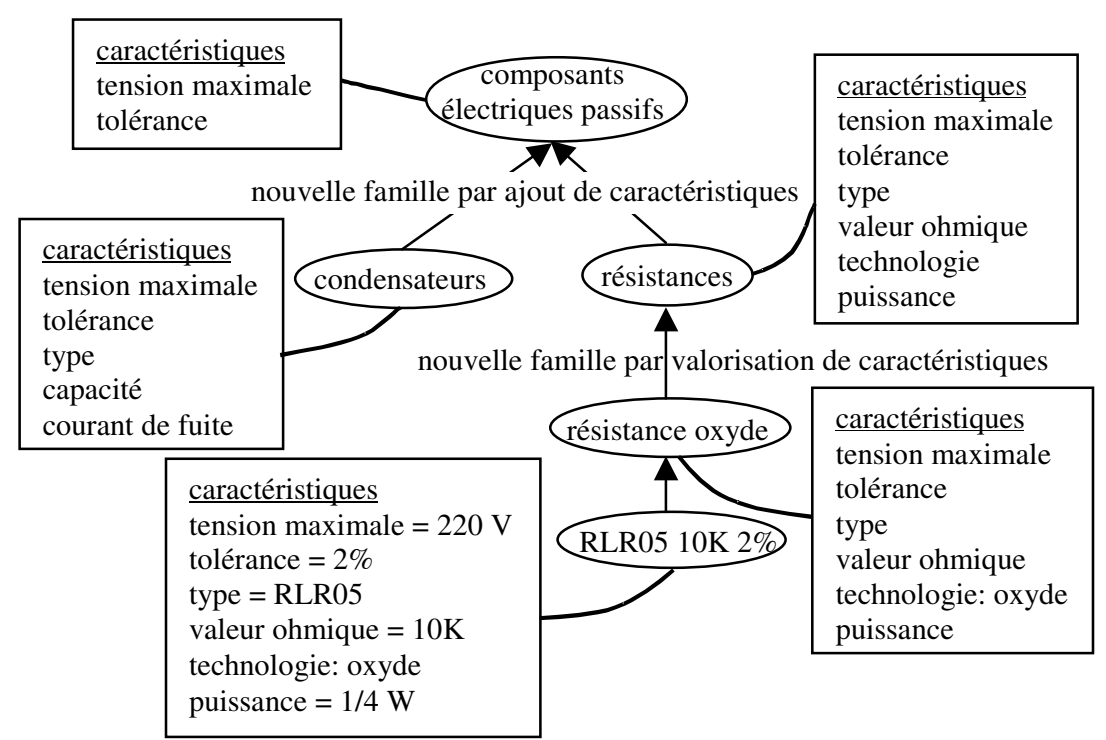

Fig. 4. Les caractéristiques des familles et des objets techniques.

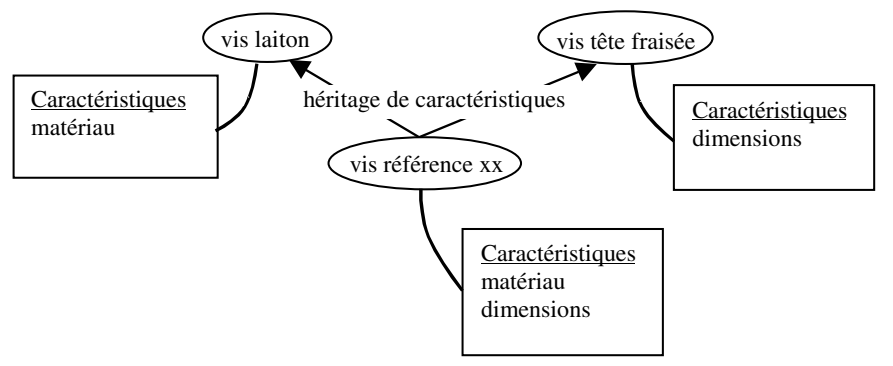

Fig. 5. L'héritage multiple de caractéristiques.

\subsubsection{Classification à facettes}

La classification à facettes provient de Ranganathan $[5,6]$. Dans le domaine de la classification bibliographique, Ranganathan a introduit en 1967 le schéma de classification à facettes qui consiste en une collection de « petites » hiérarchies de classification orthogonales les unes aux autres. L'un de ses avantages, par rapport à une classification hiérarchique classique, est qu'elle offre une meilleure compréhension de l'ensemble de données considéré en séparant mieux les diverses relations (par exemple l'héritage, mais il y en a d'autres) qui peuvent unir deux classes.

Contrairement aux autres classifications qui sont monohiérarchiques, la classification à facettes s'appuie sur des principes de division applicables à l'ensemble des objets à classer. On parle alors de « divisions analytiques », de « divisions communes » ou de «traits universels ».

Une classification à facettes se caractérise principalement par la division des termes en sous-classes, ou facettes, utilisant une seule caractéristique (ou un seul principe) de division à la fois pour produire des groupes homogènes qui s'excluent mutuellement. Tous les domaines reposent sur une structure sous-jacente de catégories fondamentales représentées dans les facettes analysées.
Cette classification est synthétique dans la mesure où elle prévoit la structuration des concepts dans une liste de sujets qui ne seraient pas cette fois énumérés du plus générique au plus spécifique et elle est analytique parce qu'elle est structurée de manière à ce que ressorte des indices de classification, la représentation de concepts simples, organisés en catégories rigoureusement établies par ce qu'on appelle l'analyse par facettes.

\section{Différents types de liens}

Afin de représenter des familles de produits, de nombreux modèles de représentation s'appuient sur des liens représentant une relation particulière entre les différents objets concernés.

Parmi ces différentes relations quelques sémantiques particulières méritent notre attention, nous allons les décrire ici succinctement.

\subsection{Lien de composition}

Maurino [1] déclare :

« La structure du produit représente les niveaux successifs de décomposition du produit en objets techniques; elle est constituée des liens de composition (également appelés liens composécomposant) unissant un objet technique aux objets entrants dans sa composition. »

Le lien de composition a pour rôle principal de décrire une relation : «X est un composant de $\mathrm{Y} »$. Cela permet entre autre de décrire les assemblages. Généralement, les liens de composition sont porteurs d'un ensemble de propriétés supplémentaires permettant de décrire plus précisément la relation de composition (cf. Fig. 6 tirée de [1]). 


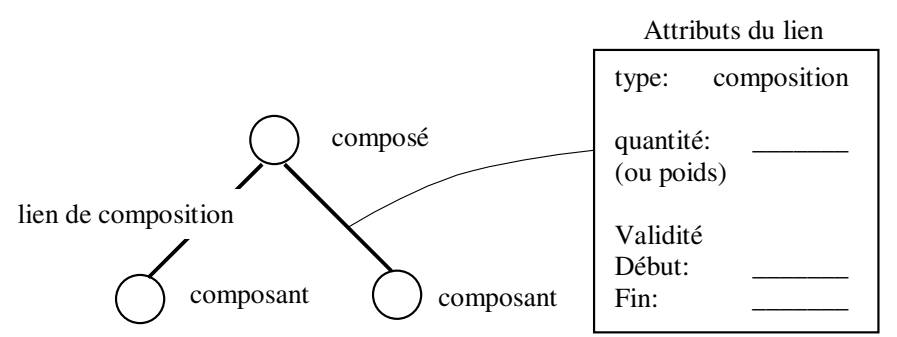

Fig. 6. Le lien de composition.

Les propriétés suivantes apparaissent très souvent :

- identification du lien : nature (composition), type de structure (fonctionnelle, technique, industrielle, ...), type de configuration décrite (de référence, produite, en service, ...),

- la quantité de composants entrant dans le composé (pour un lien entre article ou entre exemplaires), elle peut être exprimée en unité, volume, masse, ...

- une relation d'ordre permettant de trier des différents liens d'un assemblage,

- une liste de positionnement (repère topologique, matrices de positionnement, ...) permettant de localiser géographiquement des composants dans un assemblage,

- la validité du lien (date de création, date de péremption, ...).

De nombreuses autres sémantiques sont utilisées.

\subsection{Lien d'interface}

Les liens d'interface associent de façon transversale plusieurs objets techniques au sein d'une même structure du produit (Fig. 7). Ces liens permettent de :

- formaliser les relations de dépendance entre fonctions ou entre organes,

- exprimer la compatibilité entre différents articles pour un produit,

- pondérer la dépendance relative des deux objets associés par le lien.

Ce type de lien permet de représenter les contraintes existantes entre différents composants ou assemblages par exemple.

\subsection{Liens inter points de vue}

Le travail présenté par Harani [7] avait pour but de capitaliser les connaissances liées à la description du produit et celles liées au déroulement de son processus de conception. Pour cela, deux modèles ont été proposés : un modèle produit et un modèle processus de conception.

Le modèle produit proposé (Fig. $8^{3}$ ) est de type nomenclature de composition et contient deux points de vue

\footnotetext{
${ }^{3}$ Dans ces différents modèles, Harani a utilisé la modélisation Entité-Association.
}

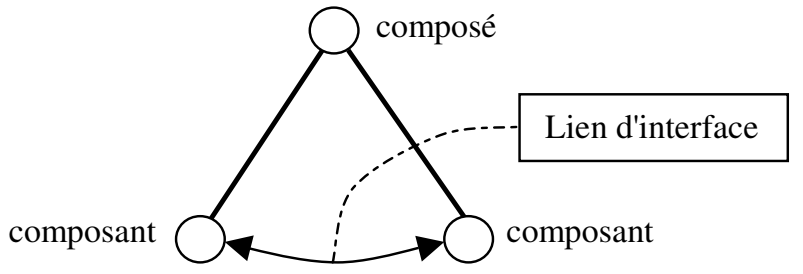

Fig. 7. Le lien d'interface.

(structurel et fonctionnel), afin de permettre deux approches complémentaires (approche produit et approche métier).

De manière à rendre l'opération de consultation des informations attachées à un produit possible, il a été choisi d'utiliser les points de vue. En effet, cette notion a pour principal objectif la description d'une entité complexe ayant plusieurs facettes. Ainsi une multitude de « grappes» d'information peuvent être définies. Les points de vue permettent :

- de structurer la connaissance relative au produit en blocs homogènes (gestion de la complexité),

- d'enrichir la spécification technique du produit en définissant de nouveaux points de vue si nécessaire,

- d'établir des liens entre points de vue assurant ainsi la complémentarité des points de vue.

En résumé, les points de vue permettent de structurer l'information (à partir de différents critères soit métiers, soit produits) pour la rendre plus représentative, plus compréhensive et donc exploitable.

Le travail d'Harani part des postulats suivants :

- tout point de vue peut être représenté à l'aide d'une structure de graphe,

- chaque graphe est constitué par un ensemble de nœuds et de liens entre nœuds,

- les nœuds sont destinés à renfermer des informations. Ces informations peuvent être des noms de paramètres, de descriptions, de variables d'où la nécessité de définir une entité nœud (Fig. 9),

- les nœuds sont reliés entre eux par des liens. Les liaisons ne sont pas forcément d'une cardinalité 1-1 mais peuvent parfois être de l'ordre de $1-n$ ou même $n-m$ entre les nouds,

- les liens entre les nœuds sont particuliers car ils sont porteurs d'une sémantique (Fig. 10). En effet, deux nœuds peuvent être reliés entre eux pour différentes raisons : pour exprimer un lien de composition, de spécialisation, d'appartenance, ou autre. Plusieurs types de liens sont donc nécessaires,

- les liens ne concernent pas uniquement l'établissement de liaisons entre nœuds d'un même graphe et donc d'un même point de vue. En effet, l'apport sémantique attaché aux liens leur permet d'exprimer aussi une relation entre nouds de deux ou plusieurs graphes différents. Il est alors possible d'exprimer un lien tel que, par exemple, l'équivalence fonctionnelle spécifiant qu'il s'agit d'un même nœud perçu différemment d'un point de vue à l'autre. 


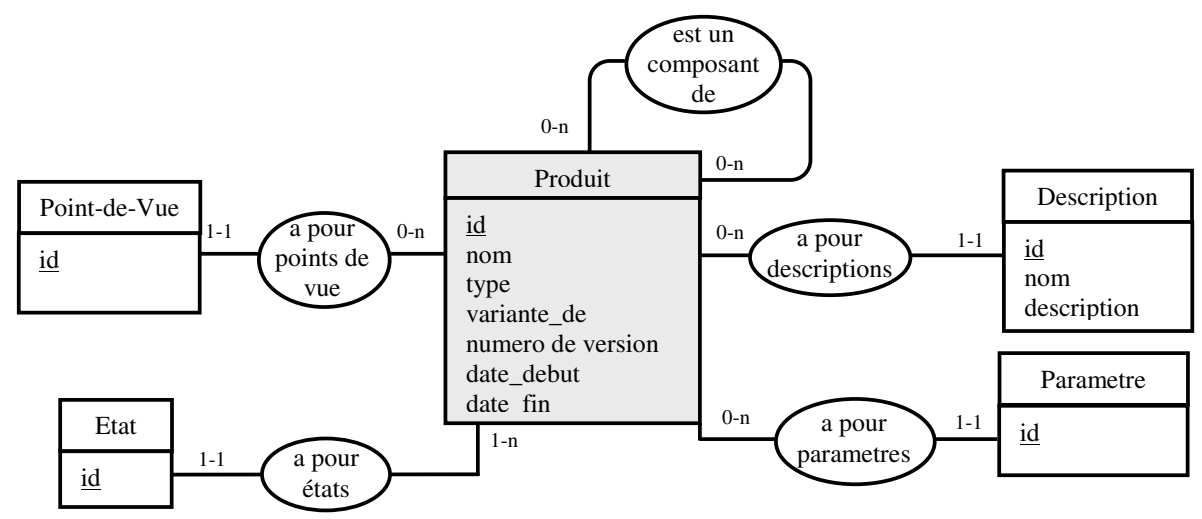

Fig. 8. Méta-modélisation du concept produit.

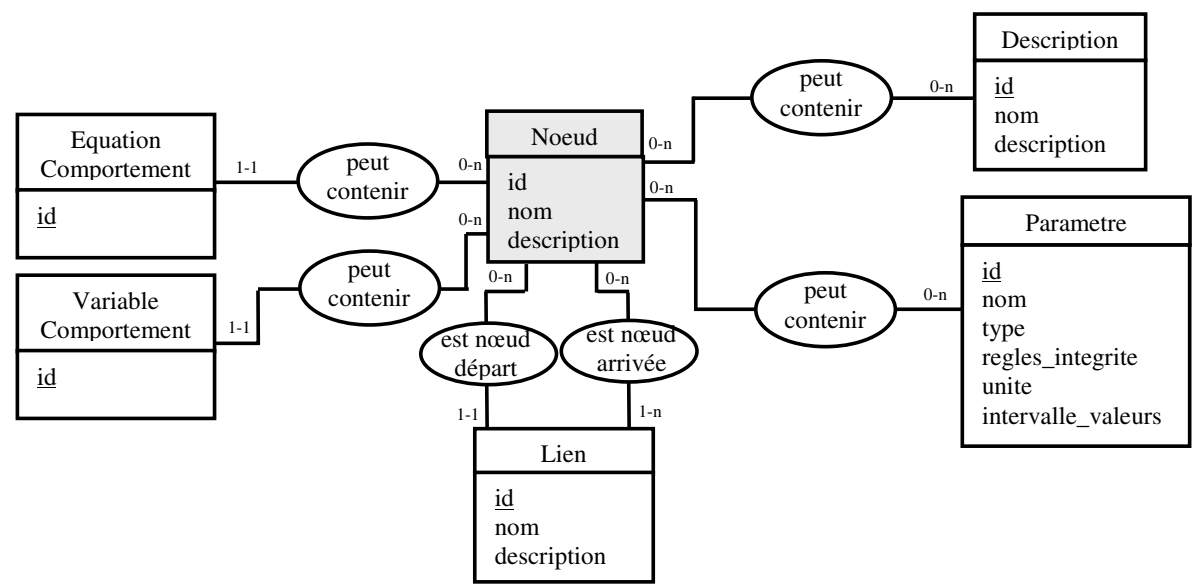

Fig. 9. Méta-modélisation de l'entité nœud.

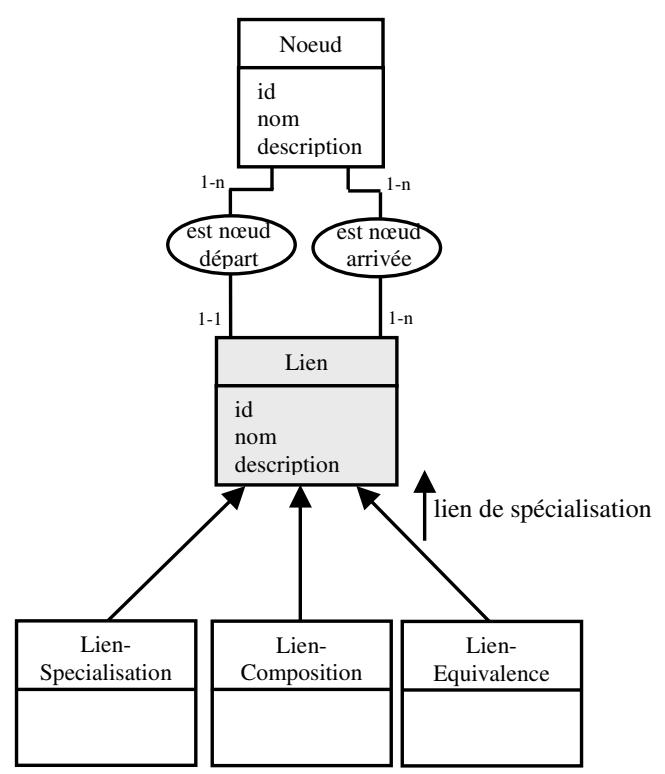

Fig. 10. Méta-modélisation de l'entité lien.
Tableau 2. Cardinalités sur relations.

\begin{tabular}{ll}
\hline $1 . . \mathrm{n}$ & le lien a un caractère obligatoire (variantes) \\
\hline $0 . . \mathrm{n}$ & le lien est optionnel (options) \\
\hline
\end{tabular}

\subsection{Contraintes inter-liens}

Sellini [8] propose d'utiliser des cardinalités sur les relations ainsi que des connecteurs sur relation. Bien que ce travail ne soit pas dédié à la représentation de la diversité mais à la conception, les cardinalités (Fig. 12) permettent de distinguer les options et variantes (Tab. 2), alors que les connecteurs sur relation (Fig. 13) permettent de poser des contraintes entre les différents composants.

Quatre sémantiques sont définies pour les relations inter-liens (contraintes) et explicités dans le tableau 3.

Le modèle inclut un mécanisme de vérification de la cohérence de l'ensemble et de simplification du modèle, d'interdiction, de fusion, de non redondance, de non sens et d'incohérence.

\section{Modélisation de la diversité}

Sont présentés ici des modèles génériques de familles de produits ainsi que des modèles de configuration.
Un exemple fourni figure 11 concerne la représentation d'un circuit d'éclairage. 


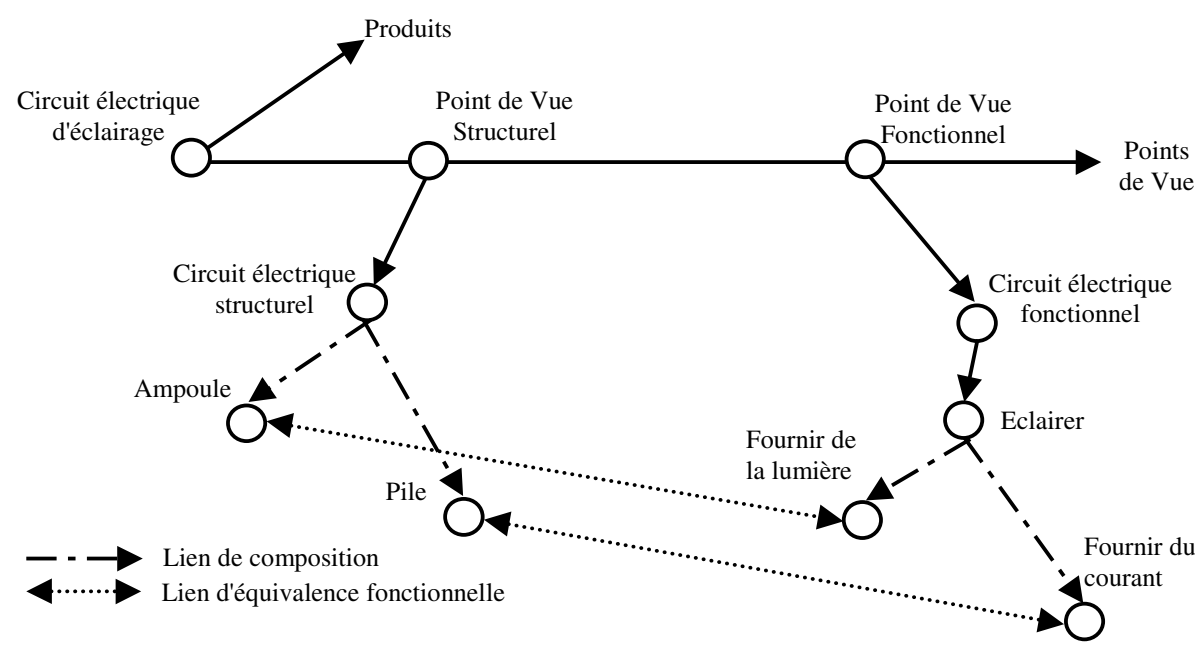

Fig. 11. Liens inter et intra points de vue d'un circuit électrique d'éclairage.

Tableau 3. Sémantique inter-liens.

\begin{tabular}{ccl}
\hline Connecteur & Symbole & Définition \\
\hline ET-Equivalent & + & $\begin{array}{l}\text { A est nécessairement composé de B, de C } \\
\text { et de D }\end{array}$ \\
\hline Au moins 1 & $1+$ & $\begin{array}{l}\text { A nécessairement composé d'au moins un élément B, } \\
\text { ou C, ou D, ou les deux, ou les trois. }\end{array}$ \\
\hline Exclusion mutuelle & $*$ & $\begin{array}{l}\text { A nécessairement composé d'un seul des éléments. } \\
\text { Le choix de B interdit C et D. }\end{array}$ \\
\hline Implication & $\rightarrow$ & $\begin{array}{l}\text { Selon le sens de l'implication, le choix de C entraine } \\
\text { obligatoirement l'existence de B mais B peut exister sans C }\end{array}$
\end{tabular}

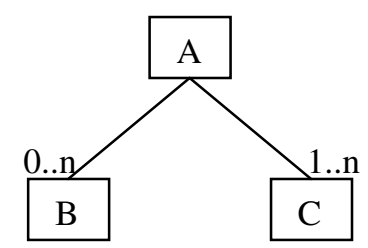

Fig. 12. Cardinalités sur relations.

\subsection{Modèles génériques de familles de produit}

Une modélisation en familles de produits permet de structurer la diversité. Les familles de produits permettent d'augmenter la variété commerciale en limitant les efforts de développement, fabrication et maintenance. Les familles de produit sont basées sur la technique de réutilisation.

\subsubsection{Arbres de classification}

O'Donnel et al. [9] proposent une méthodologie a posteriori qui vise principalement à ranger la production existante, dans une petite entreprise, de manière à concevoir un produit nouveau à partir des composants standards utilisés dans les autres produits. Il s'agit de

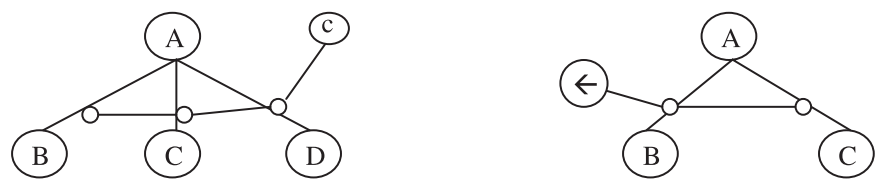

Fig. 13. Sémantique des connecteurs.

lier deux modèles pour représenter une famille de produit générique en croisant un modèle d'arbre de classification de famille de produit (PFCT : Product Familly Classification Tree) : arbre «a kind of » avec une nomenclature (PBS : Product Breakdown Structure) : arbre «a part of $»$.

Une méthodologie de structuration de produit permet d'aider les concepteurs à gérer le processus de définition de configuration d'un nouveau produit. Le processus de configuration doit être itératif et doit permettre un changement de décision. De plus, l'information du produit doit être gérable c'est-à-dire les composants, relations et autres données produites doivent être organisés de manière à permettre un accès et une manipulation facile. Enfin, la configuration des solutions doit être uniforme, c'est-à-dire que le travail de gestion de configuration est de maintenir cette uniformité entre les décisions et les éléments sélectionnés. 


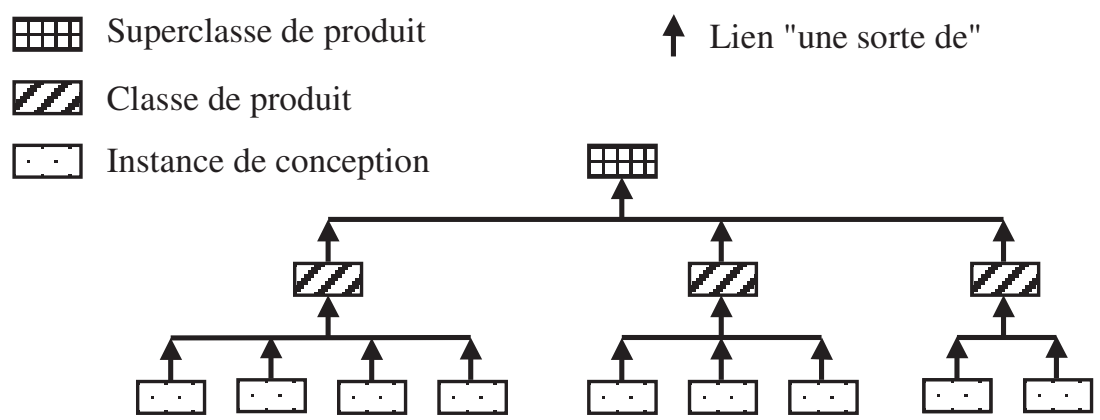

Fig. 14. Arbre de classification des familles de produits.

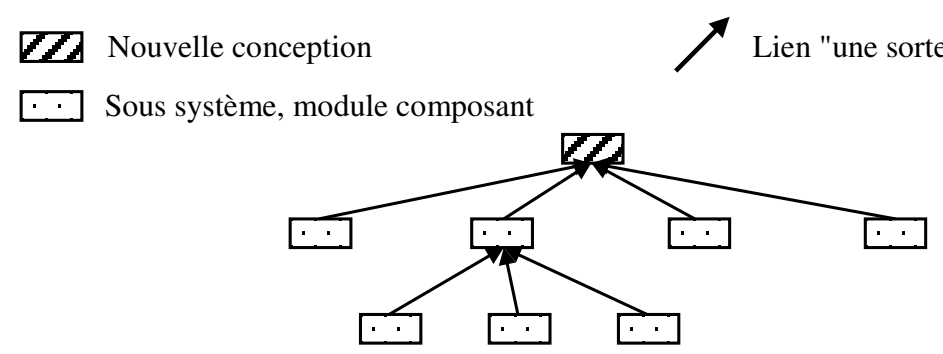

Fig. 15. Nomenclature structurelle.

\section{Modélisation des connaissances sur la structure produit}

Le but principal est de produire une nomenclature pour un nouveau produit qui soit une combinaison d'éléments sélectionnés parmi une série de décisions de conception. Les éléments d'une nomenclature peuvent être spécifiques ou abstraits. Les éléments abstraits peuvent être spécifiés plus tard par un module ou un soussystème une fois qu'il sera prêt à être spécifié.

\section{Arbres de classification de famille de produit}

Une étendue de produits peut être rangée dans un arbre de classification de famille de produit (cf. Fig. 14).

- Chaque nœud de l'arbre représente un produit ou une classe de modules avec ses composants.

- Les relations entre deux classes sont de type : «une sorte de»(«a kind of »). Chaque classe d'entité a son propre PFCT. L'association d'une nomenclature abstraite avec un nœud du PFCT distingue ce type d'arbre des autres familles d'arbres.

Deux types de contraintes de conception peuvent être représentées :

- L'une représente les dépendances le long du PFCT.

- L'autre représente un ensemble de limitation des combinaisons possibles pour les sous systèmes, composants, éléments.

Exemple de dépendance logique entre les arbres des familles Heater et Motor:

\section{IF Direct Heater or Indirect Heater is chosen. THEN Small Motor will be chosen for its Motors part.}

On peut utiliser les relations AND, OR et NOT pour réduire l'ensemble des possibles.
Le PBS représente une liste d'éléments dans une structure hiérarchique (Fig. 15). Il faut noter la différence de signification des liens « a part of » et « a kind of ». Tous les attributs, caractéristiques et propriétés des éléments sélectionnés sont enregistrés dans la liste des éléments. Le $\mathrm{PBS}$ est représenté par un arbre hiérarchique «ET».

\section{Méthodologie de description}

Le but est de fournir un modèle de représentation de structure de produits variés pour un produit existant. La méthodologie se compose de quatre étapes:

1. Créer le PFCT : Regrouper les produits en catégories, le PFCT représente l'étendue et la variété des produits.

2. Réaliser le PBS : Le but est d'analyser la structure de chaque produit.

3. Comparer les PBS : Identifier la variété, approche modulaire, matrice de comparaison.

4. Créer un modèle produit générique.

\subsubsection{Langage de programmation}

Olsen et al. [10] proposent une procédure orientée nomenclature de produit générique.

L'approche conventionnelle est de représenter une nomenclature pour chaque variante de produit, ce qui est impossible quand le nombre de variantes devient trop important. Il est préférable de décrire et de maintenir une seule structure, générique, qui contient toutes les variantes d'un produit. Cette structure pourra alors être utilisée pour générer la nomenclature de chaque variante particulière. 
Tableau 4. Exemple de modélisation générique.

\begin{tabular}{|c|c|}
\hline Component $\S 200$ is & $\underline{\text { contraindre un élément }}$ \\
\hline$-\operatorname{Name}(« \operatorname{stool} ») ;$ & Body $\S 200$ is \\
\hline End component ; & $\begin{array}{l}\text { - Include } \S 400 ; \\
\text { - Constrain } \S 400 \text { with }\end{array}$ \\
\hline Body $\S 200$ is & $--\operatorname{color}($ red $)$ \\
\hline - Include $\S 400$; & - End constrain ; \\
\hline - Include $\S 500$ & - Include $\S 500$; \\
\hline End body ; & End body ; \\
\hline \multicolumn{2}{|l|}{ Component $\S 400$ is } \\
\hline \multicolumn{2}{|l|}{$-\operatorname{Name}(\ll$ seat $») ;$} \\
\hline \multicolumn{2}{|l|}{ - Seatcolor(red-blue-white); } \\
\hline \multicolumn{2}{|l|}{ - Texture(wool-vinyl-leather); } \\
\hline End component; & \\
\hline
\end{tabular}

Leur proposition est une structure générique basée sur une notation de langage de programmation. Ceci permet de décrire l'ensemble des variantes possibles pour un produit par manipulation des relations structurelles et fonctionnelles entre les composants. L'utilisateur peut construire n'importe quelle variante de produit. Les spécifications sont données dynamiquement pendant que la nomenclature générique est exécutée.

Un exemple de modélisation est donné dans le tableau 4.

Cette modélisation signifie que le composant $\S 200$ est une chaise («stool»), qu'il est constitué des composants $\S 400$ et $\S 500$. Le composant $\S 400$ est l'assise du siège («seat »), il peut être rouge, bleu ou blanc et est constitué de laine, de vynil ou de cuir. Le langage permet de contraindre un élément, dans l'exemple le composant $\S 400$ est contraint à « rouge ».

Le modèle est bien sûr plus complet et intègre des branchements conditionnels (Case $§ 200$ is... End case ;), le versionnement (Component $\$ 400$ is... versionNumber(1-2-3-4); ... End component ;), il est aussi possible de définir des valeurs par défaut, ...

\subsection{Modèles de configurations}

Tiihonen et al. [11] ont réalisé une analyse à partir de 10 cas industriels qui montre que les industriels sont sensibilisés au problème de configuration de produit. Ils notent que les entreprises manquent de méthodes et d'outils pour représenter les modèles. Les nomenclatures génériques et structures équivalentes ne sont pas utilisées.

Certaines entreprises ont testé les configurateurs de produit, mais ne les utilisent pas quotidiennement. Elles souhaitent un outil pour les aider mais pas quelque chose de totalement automatique qu'elles ne maîtrisent pas.

À travers un ensemble de travaux [11-16], le Product Data Management Group (T. Männisto, R. Sulonen, H. Peltonen, J. Tiihonen, T. Soininen, T. Lehtonen et A. Pulkkinen), propose une structure de produit générique

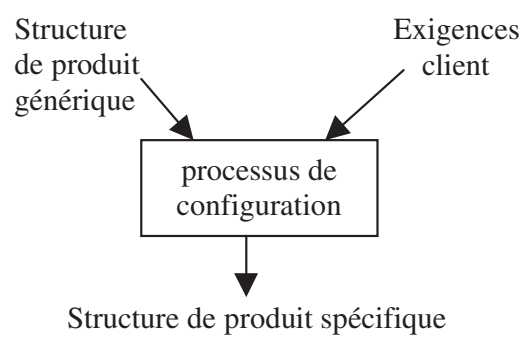

Fig. 16. Processus de configuration.

(SPG) à laquelle s'ajoute un modèle de configuration. Leur SPG représente l'ensemble des alternatives, elle se compose d'un modèle explicite et d'un modèle implicite :

- Le modèle explicite contient les composants, la hiérarchie, les options et variantes.

- Le modèle implicite contient les connaissances sur les compatibilités entre les composants et les contraintes.

À ce modèle, s'ajoute un modèle de configuration qui contient les exigences des clients (mapping des spécifications techniques exprimées dans un langage compréhensible dans le process de configuration). Ils définissent un processus de configuration (Fig. 16) qui à partir de la SPG et des exigences client fournit une structure de produit spécifique.

Pour représenter un produit configurable, une nomenclature de produit ordinaire est étendue par des parties optionnelles et alternatives ainsi que par des composants paramétrables.

Un produit configurable est une famille de produits, il s'agit pour eux d'un ensemble de produits appelés les variantes d'un produit configurable. Chaque variante est construite individuellement pour chaque consommateur.

Le processus de configuration est une évolution du modèle de configuration (produit générique) vers une configuration spécifique (produit spécifique) qui représente une seule variante à travers les choix du consommateur parmi l'ensemble des configurations valides du modèle de configuration (Fig. 17).

Le modèle de configuration décrit l'ensemble des variantes possibles et décrit comment créer une variante appropriée à une spécification du consommateur. Il se compose d'une structure explicite à laquelle s'ajoutent des contraintes.

Trois niveaux de configurations sont définis $[15,16]$ :

- « configuration solution knowledge » décrit une configuration. Deux sous cas sont possibles :

- la configuration est incomplète, elle décrit alors un ensemble de produits individuels possibles,

- la configuration est spécifique et décrit une variante unique.

- « configuration model knowledge » décrit l'ensemble des configurations correctes,

- « requirement knowledge » décrit les besoins de la configuration à construire.

Un exemplaire est une description individuelle, correspondant à un unique exemplaire physique et contenant 


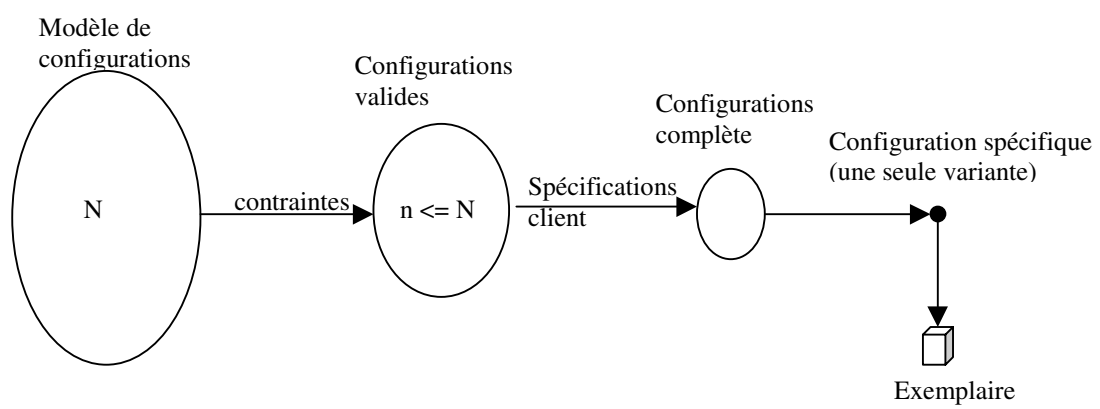

Fig. 17. Évolution du modèle de configuration.

des informations supplémentaires nécessaires et créées pendant le processus de fabrication. C'est à ce niveau que se situe la reconfiguration. Il y a reconfiguration lorsque les composants évoluent indépendamment des produits dans lesquels ils interviennent.

\section{STEP}

La norme $\mathrm{STEP}^{4}$ est une norme internationale de l'ISO $^{5}$ référencée ISO 10303 [17] et subdivisée en sous-parties. Elle est développée au sein du groupe ISO/TC184/SC4 ${ }^{6}$. Le but est la construction d'un standard permettant de traiter la représentation et l'échange de modèles de produits en couvrant tout leur cycle de vie.

La norme STEP s'appuie sur un modèle en trois couches représenté figure 18 :

- le modèle utilisateur décrit ce que voit l'utilisateur,

- le modèle informatique représente la structure de données,

- le modèle physique est la base de données.

L'origine de cette normalisation vient d'un besoin industriel d'échange de données entre les différentes applications informatiques intervenant tout au long du cycle de vie du produit, ainsi que dans un but d'archivage à long terme $[18,19]$.

Partant de ces objectifs, l'intérêt porte ici sur la manière dont STEP permet de modéliser des structures de produits génériques. Pour cela, deux protocoles d'application (AP 203 et AP 214), applicables aux produits mécaniques, sont considérés. Les protocoles d'application préconisent un emploi pour la norme dans un contexte particulier, ils définissent des méthodes d'implémentation pour l'échange et la manipulation des informations.

\subsection{STEP-AP 203}

Le protocole d'application 203 [20] se focalise sur le domaine de la conception 3D d'ensembles mécaniques avec gestion de configuration. Il a pour objectif de couvrir les

\footnotetext{
${ }^{4}$ STEP : STandarts for Exange of Product model data.

5 ISO : International Organization for Standardization.

${ }^{6}$ http://www.nist.gov/sc4/
}

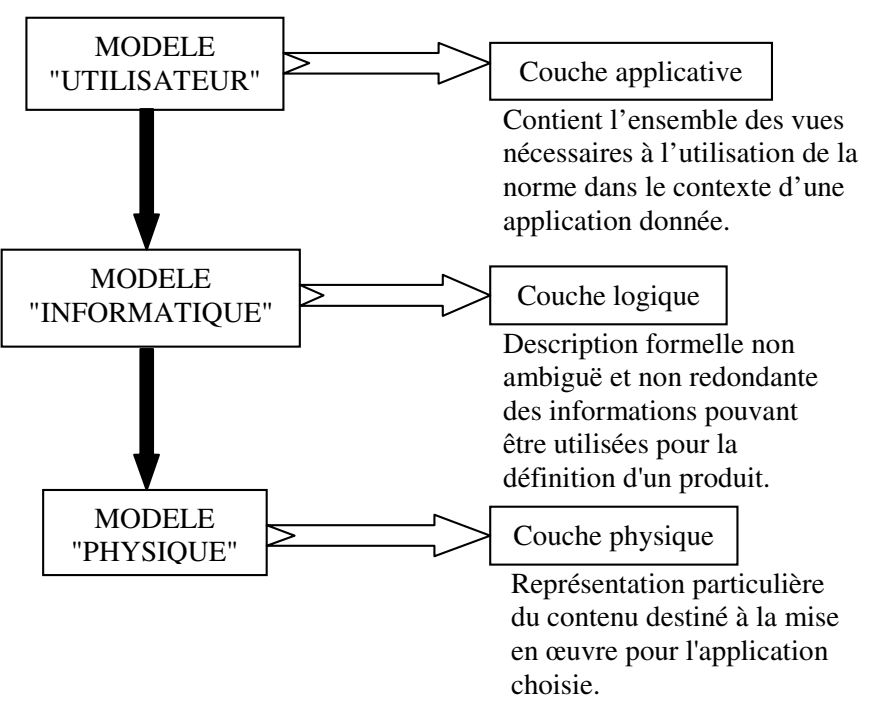

Fig. 18. Modèle en trois couches de STEP.

données associées à un produit lors de sa phase de conception, ceci concerne les données de type géométrique mais aussi la définition et le contrôle de la configuration de ces produits.

La norme considère les trois critères d'abstraction suivants (chacun pouvant être appliqué de manière récursive) :

- la classification qui consiste à regrouper les objets en fonction de leurs propriétés. Des classes sont ainsi fabriquées, dont on essaie d'obtenir qu'elles soient, pour un même niveau, exclusives, exhaustives et homogènes de manière à éviter les ambiguïtés d'appartenance. Cette notion conduit souvent à des hiérarchies,

- la décomposition qui consiste à relier un objet à ses composantes ou parties élémentaires. Inversement l'agrégation consiste à recomposer des éléments simples pour obtenir un objet plus complexe. Appliquée récursivement, la décomposition revient à affiner un élément par approches successives,

- la généralisation qui consiste à relier les objets semblables à un autre objet plus général de niveau plus élevé. Elle revient à élargir le champ du modèle, le but étant de minimiser les informations à stocker, les propriétés associées au type d'objet le plus général étant alors héritées par les types plus spécialisés. 


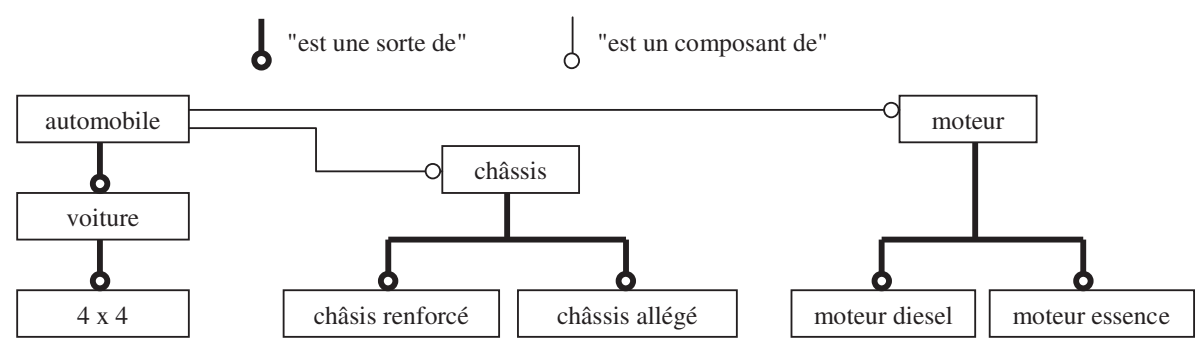

Fig. 19. Modélisation de produits génériques avec STEP-AP 203.

Inversement la catégorisation a pour but de séparer les objets en différents groupes selon certains critères.

La description des structures de données couvre deux types de données :

- les pièces (les données géométriques, créées par un outil de CFAO),

- les assemblages (les données de structuration de produit et de gestion de configuration).

Elle permet la structuration par domaine et par technologie («vues »), le modèle proposé gère :

- les nomenclatures avec options et variantes,

- les versions,

- la traçabilité (diffusions, modifications, validités),

- le workflow (approbations, spécifications des clients, certifications, confidentialité, ...).

Männistö et al. [21] étudient la modélisation STEP afin de modéliser des structures de produits génériques. Ces méthodes sont importantes pour de nombreux produits complexes, parmi lesquels les produits configurables sont un exemple. Ils notent deux types de liens qui permettent de décrire des produits non complètement instanciés. Le lien « est une sorte de » permet la classification et donc le partage d'information à travers l'héritage. Le lien « est un composant de » quant à lui permet de décrire les assemblages.

Une structure de produit explicite qui s'appuie sur les deux types de liens énoncés et supporte les éléments optionnels «optional part» et les variantes «alternative part » est complétée par une structure de produit implicite qui donne les conditions supplémentaires qui doivent être respectées par un produit valide. Par exemple la structure de produit implicite peut interdire la combinaison de certaines options. Cette condition peut être exprimée par une règle $\mathrm{EXPRESS}^{7}$ comme celle ci :

$\mathrm{NOT}($ option 1 IN «types of components $(S E L F)$ » $\mathrm{AND}$ «option $2 » \mathrm{IN}$ «types of components $(S E L F) »)$

qui signifiera que le produit considéré (« types of components $(S E L F) »)$ ne pourra contenir à la fois option 1 et option 2.

Un exemple de modélisation explicite est montré figure 19.

\footnotetext{
7 Le langage EXPRESS est une norme (ISO 10303-11 [22])
} de représentation formelle de données.

\subsection{STEP-AP 214}

Le protocole d'application 214 [23] est un protocole d'application pour l'automobile destiné à décrire l'ensemble des données techniques générées lors de l'étude d'un véhicule et de ses composants, pièces et outils de fabrication, et plus largement les produits à forte diversité fabriqués en grande série.

Dans le cas de l'industrie automobile, la combinatoire des options et variantes pour un produit devient trop importante pour permettre la représentation de toutes les nomenclatures explicites. Dans Chambole [24], la gestion de la diversité véhicule est alors présentée à travers la nomenclature variationnelle qui permet de décrire le produit avec des spécifications structurées par catégories. Une spécification peut être utilisée par plusieurs produits. Une pièce pouvant être composée d'autres pièces, il est alors possible de les structurer au moyen d'une structure explicite d'assemblage.

Les produits sont caractérisés par des attributs de conception, gestion, personnalisation, descriptifs à l'aide de l'entité «specification». Ces caractéristiques sont regroupées par classes (« category»). Par exemple, vitre électrique a pour classe VE et pour attributs 00 pour les véhicules dépourvus de vitre électrique et 01 pour ceux qui en ont.

Ainsi un produit se compose de quelques attributs de base. Ceci permet d'obtenir un titre étendu du produit sur lequel reposeront les nomenclatures des produits. Il est alors possible de définir des règles d'utilisation de ces classes/attributs. Par exemple une règle peut être :

\section{Si Pack électrique alors Vitre électrique et Fermeture centralisée,}

qui se traduit par :

\section{SI PA01 ALORS (VE01 ET FC01)}

Des attributs supplémentaires permettent de spécifier d'autres natures de contraintes au niveau de la nomenclature :

- l'attribut mandatory permet de spécifier si l'utilisation d'une classe est obligatoire (TRUE) ou optionnelle $(F A L S E)$,

- l'attribut association type permet de préciser si l'attribut d'un véhicule est monté en standard et peut être remplacé (replaceable standard), monté en standard et ne peut pas être remplacé (non replaceable standard), disponible (availability) ou optionnel (option), 


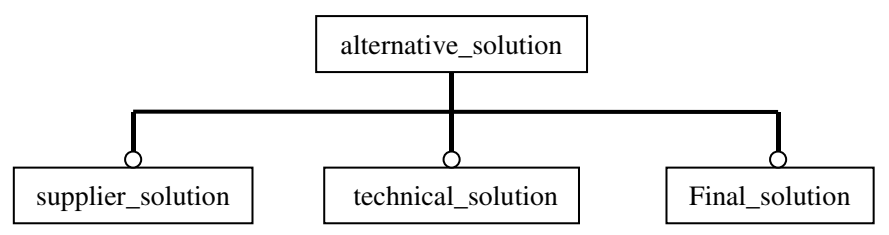

Fig. 20. Différents types de solutions.

- l'attribut implicit exclusive condition a pour rôle d'autoriser (FALSE) ou non (TRUE) le choix de plusieurs attributs dans une classe.

Afin de faciliter l'organisation et la structuration des informations et pièces d'un véhicule, deux découpages sont proposés :

- le découpage dit organique ( product component») qui est une vue structurelle du véhicule. Il s'agit d'une structure hiérarchique dont les nœuds sont des organes tels qu'une boite de vitesse.

- le découpage dit fonctionnel («product function») qui est une vue fonctionnelle du véhicule. Il s'agit aussi d'une structure hiérarchique mais les nœuds sont ici des fonctions telles que le freinage.

La diversité des solutions peut être structurée à l'aide de «alternative solution » qui peut être de différents types (cf. Fig. 20) :

- les solutions techniques («technical solution») permettent l'identification de techniques différentes remplissant un même besoin fonctionnel,

- les solutions fournisseur («supplier solution») identifient les solutions par fournisseur (ex : projecteur VALEO),

- les solutions dites finales («final solution») permettent d'identifier les solutions de bases auxquelles des caractéristiques « sensitives » ont été ajoutées (des pièces colorées par exemple).

\section{Conclusion}

À travers cette étude bibliographique concernant la représentation des familles de produits, l'auteur n'a pas cherché à être exhaustifs dans les différents modèles présentés. Le but était de montrer l'étendue des différentes représentations. Cette recherche en largeur permettra au lecteur de sélectionner un modèle général apte à représenter le problème qui le concerne.

Cependant à travers l'analyse de la littérature présentée, certains points particuliers méritent d'être mis en relief. Tout d'abord notons les principaux points de consensus :

1. Structuration : Dans les différents travaux présentés, il est apparu que la structuration des données est mieux gérée par les liens. Les liens étant porteurs d'une sémantique plus ou moins complexe.
2. Sémantique associée aux liens : Quels que soient les travaux sur la structuration des données présentés ici, les liens sont toujours porteurs d'une sémantique, c'est au niveau de cette sémantique que repose toute l'information de structuration. Il faut noter les exemples de sémantique suivants : «a part of », «a kind of », «OR», composition, spécialisation et appartenance qui sont apparus assez souvent et à travers différents travaux. Certaines modélisations proposent de faire porter aux liens les informations nécessaires à la traçabilité des évolutions, la norme STEP $[17,20,22$, 23] en est le meilleur exemple et le plus complet.

3. Familles de produit : De manière à modéliser les produits à forte diversité, les différents travaux ont proposé de regrouper les produits en fonction de leurs points communs, qui peuvent apparaître dans plusieurs vues et dépendre de critères assez différents. Après cette étape de comparaison, les produits sont rangés dans des «familles » qui permettent de partager certaines caractéristiques. Principalement, deux méthodes de représentations des familles ont été uti-

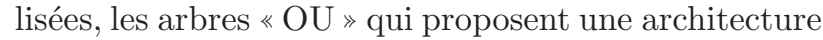
de type explicite, et une modélisation de type Objet en s'appuyant sur le concept d'héritage.

Une famille de produit identifie les points communs et les différences entre les produits individuels qui forment une gamme de produits.

Le concept de familles de produit permet donc :

- de représenter une grande variété de produits à partir d'un ensemble restreint d'éléments,

- de limiter les coûts de développement et fabrication par réutilisation d'éléments communs à tout un ensemble de produit.

Enfin, vis-à-vis du problème auquel nous nous sommes attachés, l'état de l'art montre qu'une modélisation de type famille de produit est la mieux adaptée à la représentation des variantes de produits.

Dans la modélisation des familles de produit, il faudra s'intéresser à savoir quelle est la dynamique de versionnement des liens et constituants et comment elle se propage dans les différentes nomenclatures de produits. Männistö [14] présente clairement cette problématique et catégorise différents types de versionnement selon l'étendue de la propagation du versionnement, mais il ne résout pas le problème.

De même, dans la modélisation des familles de produits, les process utilisés pour la réalisation des différents constituants et assemblages ne sont pas représentés. Il serait néanmoins intéressant d'enrichir la représentation du produit par les séquences d'assemblage, entre autre, ce qui permettrait de lier le produit et le process dès sa représentation.

\section{Références}

[1] M. Maurino, La gestion des données techniques, technologie du concurrent engineering, Collection Organisation industrielle, Masson, 1994 
[2] J. Browne, J. Haiken, J. Shivnan, Les systèmes de production dans un environnement CIM, AFNOR, 1994

[3] C. McMahon, J. Browne, CAD CAM 2nd Edition, Principles, Practice and Manufacturing Management, Addison-Wesley, 1998

[4] P. Dietz, A. Ort, The use of ISO 13584 methodology for building part hierarchies in practice, European PDT Days (1997), pp. 83-89

[5] E. Garfield, A Tribute to S.R. Ranganathan : Part 1. Life and Works, Essays of an Information Scientist, 7 (1984) $37-44$

[6] E. Garfield, A Tribute to S.R. Ranganathan : Part 2. Contribution to Indian and International Library Science, Essays of an Information Scientist, 7 (1984) 45-49

[7] Y. Harani, Une approche multi-modèles pour la capitalisation des connaissances dans le domaine de la conception, Thèse de Doctorat, Institut National Polytechnique de Grenoble, 1997

[8] F. Sellini, Contribution à la Représentation et à la vérification de modèles de connaissances produit en ingénierie d'ensembles mécaniques, Thèse de Doctorat, École centrale Paris, 1999

[9] F.J. O'Donnell, K.J. MacCallum, T.D. Hogg, B. Yu, Product structuring in a small manufacturing enterprise, Computers in Industry 31 (1996) 281-292

[10] K.A. Olsen, P. Saetre, A. Thorstenson, A procedureoriented generic bill of materials, Computers Ind. Engng, 32(1) (1997) 29-45

[11] J. Tiihonen, T. Soininen, T. Männistö, R. Sulonen, State of the practice in product configuration : A survey of 10 cases in the finnish industry, Knowledge Intensive CAD, 1 (1996) 95-114

[12] T. Männistö, H. Peltonen, R. Sulonen, View to product configuration knowledge modeling and evolution, Configuration-papers from the 1996 AAAI Fall symposium, Boi Faltings and Eugene Freuder, AAAI Press, 1996, pp. 111-118

[13] T. Männistö, R. Sulonen, Evolution of schema and individuals of configurable products. Advances in Conceptual Modeling, Lecture notes in Computer Science, 1999

[14] T. Männisto, T. Soininen, J. Tiihonen, R. Sulonen, Framework and conceptual model for reconfiguration, Proceedings of the AAAI'99 Workshop on Configuration, AAAI Technical Report, AAAI Press, 1999
[15] H. Peltonen, T. Männistö, T. Soininen, J. Tiihonen, A. Martio, R. Sulonen, Concepts for modeling configurable products. European PDT Days (1998), pp. 189-196

[16] J. Tiihonen, T. Lehtonen, T. Soininen, A. Pulkkinen, R. Sulonen, A. Riitahuhta, Modeling configurable product families. Proceedings of 4th WDK Workshop on Product Structuring, Delft University of Technology, The Netherlands, October 22-23, 1998

[17] ISO 10303-1, Industrial Automation Systems and Integration, Product Data Representation and Exchange, Part 1 : Overview and Fondamental Principles. ISO International Organization for Standardisation, Geneva, 1994

[18] S. Arbouy, A. Bezos, A.F. Cutting-Decelle, P. Diakonoff, P. Germain-Lacour, J.P. Letouzey, C. Veil, STEP Concepts Fondamentaux, AFNOR, 1994

[19] F. Chambolle, Conception de produits mécaniques, Chap. 18 : Normes et échange de données : le standard STEP, Hermes, Sous la direction de M. Tollenaere 1998, pp. $435-454$

[20] ISO 10303-203, Industrial Automation Systems and Integration, Product Data Representation and Exchange, Part 203 : Application Protocol : Configuration Control Design, ISO - International Organization for Standardisation, Geneva, 1994

[21] T. Männistö, H. Peltonen, A. Martio, R. Sulonen, Modeling generic product structures in STEP, ComputerAided Design, 30(14) (1998) 1111-1118

[22] ISO 10303-11, Industrial Automation Systems and Integration, Product Data Representation and Exchange, Part 11 : Description Methods : The EXPRESS Language Reference Manual, ISO - International Organization for Standardisation, Geneva, 1994

[23] ISO 10303-214, Industrial Automation Systems and Integration, Product Data Representation and Exchange, Part 214 : Application Protocol : Core Data for Automative Mechanical Design Processes, ISO International Organization for Standardisation, Geneva, 1998

[24] F. Chambolle, Un modèle produit piloté par les processus d'élaboration : Application au secteur automobile dans l'environnement STEP, Thèse de Doctorat, École centrale Paris, 1999 